PROCEEDINGS OF THE

AMERICAN MATHEMATICAL SOCIETY

Volume 130, Number 12, Pages 3555-3563

S 0002-9939(02)06713-8

Article electronically published on July 2, 2002

\title{
A CONSTRUCTION OF MULTIRESOLUTION ANALYSIS BY INTEGRAL EQUATIONS
}

\author{
DONG-MYUNG LEE, JUNG-GON LEE, AND SUN-HO YOON \\ (Communicated by Christopher D. Sogge)
}

\begin{abstract}
In this paper we present a versatile construction of multiresolution analysis of two variables by means of eigenvalue problems of the integral equation, for $\lambda=2$. As a consequence we show that if $\phi(x)$ is the solution of the equation $\phi(x)=\lambda \int_{\mathbb{R}} h(2 x-y) \phi(y) d y$ with supp $\hat{h}(\omega)=[-\pi, \pi]$, then $V_{j}=\operatorname{span}\left\{\phi\left(2^{j} x_{1}-k_{1}\right) \phi\left(2^{j} x_{2}-k_{2}\right) \mid k_{1}, k_{2} \in \mathbb{Z}\right\}$ constructs a two-variable multiresolution analysis.
\end{abstract}

\section{INTRODUCTION}

It is generally accepted that to research more efficiently adaptable basic wavelets used to formulate the integral wavelet transform and to establish the decomposition of elements in $L^{2}(\mathbb{R})$ in high resolution ratio requires further improvements.

In this regard, we have observed that drawing new attention to the construction of a multiresolution analysis contributed decisively to the construction of the wavelet decomposition and reconstruction in $L^{2}(\mathbb{R})$. A new and more efficient approach to making a distinction from the already established [1, 3, 7, 13, can be devoted to carrying out this procedure of improvements.

In this paper, along with our main results, we will construct the existence and solution devices of two-variable wavelet functions in $L^{2}(\mathbb{R})$ by means of an eigenvalue problem, for $\lambda=2$, of the archetypical integral equations $\phi(x)=$ $\lambda \int_{\mathbb{R}} h(2 x-y) \phi(y) d y$. Some advantages are that in contrast to the previous methods $[3,5,7$, the requirements are not as restrictive as the ones using the traditional algorithmic approach in solving wavelets in $L^{2}(\mathbb{R})$, and they allow us to uniformly describe the various earlier solution processes of one- or multivariable wavelet analysis.

\section{Preliminaries}

Throughout the paper, $L^{2}(\mathbb{R})$ will denote the Hilbert space of all Lebesgue square integrable functions on $\mathbb{R}$ with inner product $\langle f, g\rangle=\int f(x) \overline{g(x)} d x$ and norm $\|f\|=\left\{\int_{R}|f(x)|^{2} d x\right\}^{\frac{1}{2}}, \quad f, g \in L^{2}(\mathbb{R})$.

Received by the editors August 23, 2000.

2000 Mathematics Subject Classification. Primary 41A17, 42C15, 46A45 46C99.

Key words and phrases. Fourier transform, wavelet analysis, integral equation, multiresolution analysis, Riesz basis.

This paper was supported by Won Kwang University in 2002.

(C)2002 American Mathematical Society 
The signs $\wedge$ and $\vee$ denote Fourier transform and the inversion, respectively. Operators mean bounded and linear.

We recall that a multiresolution analysis is a sequence $\left(V_{j}\right)_{j \in \mathbb{Z}}$ of norm-closed subspaces of $L^{2}(\mathbb{R})$ such that

i) $V_{j} \subset V_{j+1}$.

ii) $u(x) \in V_{j}$ if and only if $u(2 x) \in V_{j+1}$.

iii) $u(x) \in V_{o}$ if and only if $u(x-k) \in V_{o}$.

iv) $\overline{\bigcup_{j \in \mathbb{Z}} V_{j}}=L^{2}(\mathbb{R})$.

v) $\bigcap_{j \in \mathbb{Z}} V_{j}=\{0\}$.

vi) There exists a function $\phi \in V_{o}$, called a scaling function, such that the system $\{\phi(x-k)\}_{k \in \mathbb{Z}}$ is a Riesz basis of $V_{o}$. That is, for all $u(x) \in V_{0}, u(x)$ have unique representation as follows: there exists $C_{k}$ such that

$$
u(x)=\sum_{k \in \mathbb{Z}} C_{k} \phi(x-k) .
$$

Moreover, there exist constants $A$ and $B$ such that

$$
A\left\|\left.u\right|_{L^{2}} ^{2} \leq \sum_{k \in \mathbb{Z}}\left|C_{k}\right|^{2} \leq B\right\| u \|_{L^{2}}^{2} .
$$

As a result of [10], a sequence $\left\{h_{k}\right\}$ exists such that the scaling function satisfies

$$
\phi(x)=\sqrt{2} \sum_{k \in \mathbb{Z}} h_{k} \phi(2 x-k) .
$$

By (2), the Fourier transform of the scaling function must satisfy

$$
\hat{\phi}(\omega)=H\left(\frac{\omega}{2}\right) \hat{\phi}\left(\frac{\omega}{2}\right)
$$

where $H(\omega)=\frac{1}{\sqrt{2}} \sum_{k \in \mathbb{Z}} h_{k} e^{-i \omega k}$.

Since $\hat{\phi}(0)=\frac{1}{\sqrt{2 \pi}}[6]$, we can apply (3) recursively. This yields, at least formally, the product formula

$$
\hat{\phi}(\omega)=\prod_{k=1}^{\infty} \hat{h}\left(\frac{\omega}{2^{k}}\right) .
$$

\section{The EXISTENCE OF WAVELETS BY INTEGRAL EQUATION}

We will now construct the existence and solution devices of two-variable wavelets in $L^{2}(\mathbb{R})$ by means of an eigenvalue problem, for $\lambda=2$, of the archetypical integral equation

$$
\phi(x)=\lambda \int_{\mathbb{R}} h(2 x-y) \phi(y) d y .
$$

In the following, we will construct the solution of $(5)$ in $L^{2}(\mathbb{R})$ by giving an appropriate restriction for $h(\cdot)$. Furthermore, we will describe two-variable wavelets $\phi(x)\left(=\phi\left(x_{1}, x_{2}\right)\right)$ from (5) by substituting $x=\left(x_{1}, x_{2}\right)^{T}, y=\left(y_{1}, y_{2}\right)^{T}$ for the solution of the equation utilizing the existence of some real scalar $\lambda(\neq 0)$.

In the sequel, $\phi(x)$ and $h(2 x-y)$ will denote $\phi(x)=\phi\left(x_{1}, x_{2}\right)$ and $h(2 x-y)=$ $h\left(2 x_{1}-y_{1}, 2 x_{2}-y_{2}\right)$, respectively, for each $x=\left(x_{1}, x_{2}\right)^{T}, y=\left(y_{1}, y_{2}\right)^{T}$.

First, we begin by giving the following theorem, clarifying the existence of the solution of (5) for the case of $\lambda=2$ by taking a suitable function $h(x)$ in $L^{2}\left(\mathbb{R}^{2}\right)$ : 
Theorem 3.1. In the case $\lambda=2$, there exists a solution of

$$
\phi(x)=\lambda \int_{\mathbb{R}} h(2 x-y) \phi(y) d y
$$

for some $h(\cdot) \in L^{2}\left(\mathbb{R}^{2}\right)$.

Proof. The Fourier transform of the above equation is

$$
\begin{aligned}
\hat{\phi}(\omega) & =\lambda \int_{\mathbb{R}}\left\{h\left[2\left(x-\frac{y}{2}\right)\right]\right\}^{\wedge} \phi(y) d y \\
& =\frac{\lambda}{2} \hat{h}\left(\frac{\omega}{2}\right) \int_{\mathbb{R}} e^{-i \omega \frac{y}{2}} \phi(y) d y \\
& =\frac{\lambda}{2} \hat{h}\left(\frac{\omega}{2}\right) \hat{\phi}\left(\frac{\omega}{2}\right)
\end{aligned}
$$

where $\omega=\left(\omega_{1}, \omega_{2}\right)^{T}$ and

$$
\hat{h}(\omega)=\frac{1}{2 \pi} \int_{\mathbb{R}} \int_{\mathbb{R}} h\left(x_{1}, x_{2}\right) e^{-i\left(\omega_{1} x_{1}+\omega_{2} x_{2}\right)} d x_{1} d x_{2} .
$$

We have the following:

$$
\hat{h}(\omega)=\left\{\begin{array}{cl}
\frac{\lambda}{2} h(\omega)(=H(\omega), \lambda=2), & \omega \in[-\pi, \pi]^{2}, \\
0, & \omega \notin[-\pi, \pi]^{2} .
\end{array}\right.
$$

From the fact that the solution of the identity (2) in Section 2 exists, the identities (2) and (5) are equivalent, which completes the proof.

The following theorem clarifies that the solution $\phi(x)$ belongs to $L^{2}\left(\mathbb{R}^{2}\right)$ :

Theorem 3.2. If the solution of $\phi(x)=\lambda \int_{\mathbb{R}} h(2 x-y) \phi(y) d y$ exists, for $\lambda=2$, and if $\prod_{k=1}^{\infty} \hat{h}\left(\frac{\omega}{2^{k}}\right)$ converges in $L^{2}\left(\mathbb{R}^{2}\right)$, then we have $\phi(x) \in L^{2}\left(\mathbb{R}^{2}\right)$.

Proof. By substituting $\lambda=2$ in (8) and replacing $\hat{h}(\omega)$ instead of $H(\omega)$ in the identity (3), we obtain the following:

$$
\begin{aligned}
\hat{\phi}(\omega) & =\hat{h}\left(\frac{\omega}{2}\right) \hat{\phi}\left(\frac{\omega}{2}\right) \\
& =\prod_{k=1}^{\infty} \hat{h}\left(\frac{\omega}{2^{k}}\right) \hat{\phi}(0) .
\end{aligned}
$$

On the other hand, since by assumption $\hat{\phi}(\omega)$ converges in $L^{2}\left(\mathbb{R}^{2}\right)$, we have the desired assertion.

Now we are ready to describe that the solution of the equation

$$
\phi(x)=\lambda \int_{\mathbb{R}} h(2 x-y) \phi(y) d y
$$

constructs the two-variable wavelet analysis.

Theorem 3.3. Let $\phi(x)$ be the solution of $(5)$, supp $\hat{h}(\omega)=[-\pi, \pi]^{2}$, and $\hat{\phi}(\omega) \neq$ $0, w \in[-\pi, \pi]^{2}$. Then $V_{j}=\operatorname{span}\left\{\phi\left(A_{j} x-k\right) \mid k^{T} \in \mathbb{Z}^{2}\right\}$ constitutes a multiresolution analysis, where $A_{j}=\left(\begin{array}{cc}2^{j} & 0 \\ 0 & 2^{j}\end{array}\right)$. 
Proof. i) From (5), we immediately have

$$
\phi(x)=\lambda \int_{\mathbb{R}^{2}} h\left(A_{1} x-y\right) \phi(y) d y .
$$

The Fourier transform of (9) is

$$
\begin{aligned}
\hat{\phi}(\omega) & =\lambda \int_{\mathbb{R}^{2}}\left\{h\left(A_{1} x-y\right)\right\}^{\wedge} \phi(y) d y \\
& =\lambda \int_{\mathbb{R}^{2}}\left\{h\left[A_{1}\left(x-A_{1}^{-1} y\right)\right]\right\}^{\wedge} \phi(y) d y \\
& =\lambda \int_{\mathbb{R}^{2}}\left|A_{1}\right|^{-1} \hat{h}\left(A_{1}^{-1} \omega\right) e^{-i \omega^{T} A_{1}^{-1} y} \phi(y) d y \\
& =\lambda\left|A_{1}\right|^{-1} \hat{h}\left(A_{1}^{-1} \omega\right) \int_{\mathbb{R}^{2}} e^{-i \omega^{T} A_{1}^{-1} y} \phi(y) d y \\
& =\lambda\left|A_{1}\right|^{-1} \hat{h}\left(A_{1}^{-1} \omega\right) \hat{\phi}\left(A_{1}^{-1} \omega\right) .
\end{aligned}
$$

If $\hat{h}(\omega)=\sum_{k \in \mathbb{Z}^{2}} h_{k} e^{-i k^{T} \omega}, \omega \in[-\pi, \pi]^{2}, k \in \mathbb{Z}^{2}$, substituting in (10), we have

$$
\hat{\phi}(\omega)=\frac{\lambda}{4} \sum_{k \in \mathbb{Z}^{2}} h_{k} e^{-i k^{T} A_{1}^{-1} \omega} \hat{\phi}\left(A_{1}^{-1} \omega\right) .
$$

By carrying out the Fourier transform of

$$
\phi(x)=\lambda \sum_{k \in Z^{2}} h_{k} \phi\left(A_{1} x-k\right),
$$

we find that (10) and (11) are equivalent.

Thus, for any $l \in \mathbb{Z}^{2}$,

$$
\begin{aligned}
\phi\left(A_{j} x-l\right) & =\lambda \sum_{k \in \mathbb{Z}^{2}} h_{k} \phi\left(A_{1} A_{j} x-A_{1} l-k\right) \\
& =\lambda \sum_{k \in \mathbb{Z}^{2}} h_{k} \phi\left(A_{j+1} x-A_{1} l-k\right) \in V_{j+1} .
\end{aligned}
$$

Hence, we obtain $V_{j} \subset V_{j+1}$.

ii) If $u(x) \in V_{j}$ and $u(x)=\sum_{k \in \mathbb{Z}^{2}} C_{k} \phi\left(A_{j} x-k\right)$, then

$$
\begin{aligned}
u\left(A_{1} x\right) & =\sum_{k \in \mathbb{Z}^{2}} C_{k} \phi\left(A_{j} A_{1} x-k\right) \\
& =\sum_{k \in \mathbb{Z}^{2}} C_{k} \phi\left(A_{j+1} x-k\right) .
\end{aligned}
$$

Thus $u\left(A_{1} x\right) \in V_{j+1}$.

iii) Suppose that $u(x) \in V_{o}$. Then $u(x)=\sum_{k \in \mathbb{Z}^{2}} C_{k} \phi(x-k)$ and

$$
\begin{aligned}
u(x-l) & =\sum_{k \in \mathbb{Z}^{2}} C_{k} \phi(x-l-k) \\
& \left.=\sum_{k \in \mathbb{Z}^{2}} C_{n-l} \phi(x-n) \text { (because } l+k=n\right) .
\end{aligned}
$$

Hence, $u(x-l) \in V_{o}$. 
iv) We prove this in two steps: first, from (11),

$$
\phi\left(A_{j} x-A_{1}^{-1} l\right)=\lambda \sum_{k \in \mathbb{Z}^{2}} h_{k} \phi\left(A_{j+1} x-l-k\right) .
$$

That is, $\phi\left(A_{j} x-A_{1}^{-1} l\right)$ is represented by the basis of

$$
\phi\left(A_{j+1} x-k\right), k \in \mathbb{Z}^{2} .
$$

Thus,

$$
\left\{\phi\left(A_{j} x-A_{1}^{-1} k\right) \mid j, k \in \mathbb{Z}^{2}\right\} \subset\left\{\phi\left(A_{j+1} x-k\right) \mid j, k \in \mathbb{Z}^{2}\right\} .
$$

Conversely, since $\left\{\phi\left(A_{j+1} x-k\right) \mid j, k \in \mathbb{Z}^{2}\right\} \subset\left\{\phi\left(A_{j} x-A_{1}^{-1} k\right) \mid j, k \in \mathbb{Z}^{2}\right\}$ is clearly satisfied, we consequently have the following:

$$
\left\{\phi\left(A_{j+1} x-k\right) \mid j, k \in \mathbb{Z}^{2}\right\}=\left\{\phi\left(A_{j} x-A_{1}^{-1} k\right) \mid j, k \in \mathbb{Z}^{2}\right\} .
$$

Second, we show that $\left\{\phi\left(A_{j} x-A_{1}^{-1} k\right) \mid j, k \in \mathbb{Z}^{2}\right\}$ is complete on $L^{2}\left(\mathbb{R}^{2}\right)$. For any $u \in L^{2}\left(\mathbb{R}^{2}\right)$, suppose that

$$
\left\langle u(x), \phi\left(A_{j} x-A_{1}^{-1} k\right)\right\rangle_{L^{2}\left(\mathbb{R}^{2}\right)}=0,
$$

that is, assume that

$$
\left\langle\hat{u}(\omega),\left\{\phi\left(A_{j} x-A_{1}^{-1} k\right)\right\}^{\wedge}\right\rangle_{L^{2}\left(\mathbb{R}^{2}\right)}=0 .
$$

Accordingly, we have

$$
\int_{\mathbb{R}^{2}} \hat{u}(\omega) \overline{\left\{\phi\left(A_{j} x-A_{1}^{-1} k\right)\right\}^{\wedge}} d \omega=\int_{\mathbb{R}^{2}} \hat{u}(\omega)\left(\left|A_{j}\right|^{-1}\right) \overline{\hat{\phi}}\left(A_{j}^{-1} \omega\right) e^{i k^{T} A_{1}^{-1} A_{j}^{-1} \omega} d \omega .
$$

Now, we put $t=A_{j}^{-1} \omega$

$$
\int_{\mathbb{R}^{2}} \hat{u}\left(A_{j} t\right) \overline{\hat{\phi}}(t) e^{i k^{T} A_{1}^{-1} t} d t=0 .
$$

Then, by (6), since supp $\hat{\phi}(\omega)=[-2 \pi, 2 \pi]^{2}$ holds, we have

$$
\begin{aligned}
& \int_{\mathbb{R}^{2}} \hat{u}(\omega) \overline{\left\{\phi\left(A_{j} x-A_{1}^{-1} k\right)\right\}^{\wedge}} d \omega \\
& =\int_{[-2 \pi, 2 \pi]^{2}} \hat{u}\left(A_{j} \omega\right) \overline{\hat{\phi}}(\omega) e^{i k^{T} A_{1}^{-1} A_{j}^{-1} \omega} d \omega=0, j, k=\mathbb{Z}^{2} .
\end{aligned}
$$

Thus

$$
\hat{u}\left(A_{j} \omega\right) \overline{\hat{\phi}}(\omega)=0, \quad \omega \in[-2 \pi, 2 \pi]^{2} .
$$

Also, by (6) and the continuity of $\hat{\phi}(\omega)$, we have

$$
\hat{\phi}(\omega) \neq 0, \quad \omega \in[-2 \pi, 2 \pi]^{2} .
$$

Consequently, $\hat{u}\left(A_{j} \omega\right)=0, \omega \in[-2 \pi, 2 \pi]^{2}$,

$$
\text { i.e., } \hat{u}(w)=0, \quad w \in\left[-2^{j+1} \pi, 2^{j+1} \pi\right]^{2} .
$$

On the other hand, since $j$ is arbitrary, we conclude that

$$
\hat{u}(\omega)=0, w \in \mathbb{R}^{2} \text {, i.e., } u(x)=0, x \in \mathbb{R}^{2} .
$$

Therefore, $\overline{\bigcup_{j \in \mathbb{Z}^{2}} V_{j}}=L^{2}\left(\mathbb{R}^{2}\right)$. 
v) We now show that $\bigcap_{j \in \mathbb{Z}^{2}} V_{j}=\{0\}$. For all $\lambda(x) \in \bigcap_{j \in \mathbb{Z}^{2}} V_{j}$, we let

$$
\lambda(x)=\sum_{k \in \mathbb{Z}^{2}} C_{j k} \phi\left(A_{j} x-k\right),
$$

and applying the Fourier transform, then

$$
\begin{aligned}
\hat{\lambda}(\omega) & =\left|A_{j}\right|^{-1} \sum_{k \in \mathbb{Z}^{2}} C_{j k} \hat{\phi}\left(A_{j}^{-1} \omega\right) e^{-i k^{T} A_{j}^{-1} \omega} \\
& =\left|A_{j}\right|^{-1} \hat{\phi}\left(A_{j}^{-1} \omega\right) f\left(A_{j}^{-1} \omega\right),
\end{aligned}
$$

where $f\left(A_{j}^{-1} \omega\right)=\sum_{k \in \mathbb{Z}^{2}} C_{j k} e^{-i k^{T} A_{j}^{-1} \omega}$.

In this case, if we take $j$ a sufficiently large negative integer, then $\hat{\phi}\left(A_{j}^{-1} w\right)=0$ is satisfied, so we have $\hat{\lambda}(w)=0$, which implies $\lambda(x)=0$.

vi) Finally, we need to prove only that $\phi(x-k), k \in \mathbb{R}^{2}$, forms a Riesz basis.

Let $u(x)=\sum_{k \in \mathbb{Z}^{2}} C_{k} \phi(x-k)$, for all $u(x) \in V_{o}$. We construct the Fourier transform

$$
\hat{u}(\omega)=H(\omega) \hat{\phi}(\omega),
$$

where $H(\omega)=\sum_{k \in \mathbb{Z}^{2}} C_{k} e^{-i k^{T} \omega}$.

On the other hand, we consider

$$
\begin{aligned}
\|u\|^{2} & =\|\hat{u}\|^{2}=\int_{\mathbb{R}^{2}}|\hat{u}(\omega)|^{2} d \omega \\
& =\int_{\mathbb{R}^{2}}|H(\omega)|^{2}|\hat{\phi}(\omega)|^{2} d \omega \\
& =\sum_{k \in \mathbb{Z}^{2}} \int_{2 k_{1} \pi}^{2\left(k_{1}+1\right) \pi} \int_{2 k_{2} \pi}^{2\left(k_{2}+1\right) \phi}|H(\omega)|^{2}|\hat{\phi}(\omega)|^{2} d \omega \\
& =\sum_{k \in \mathbb{Z}^{2}} \int_{[0,2 \pi]^{2}}|H(t+D k)|^{2}|\hat{\phi}(t+D k)|^{2} d t,
\end{aligned}
$$

where $\omega-D k=t, D=\left(\begin{array}{cc}2 \pi & 0 \\ 0 & 2 \pi\end{array}\right)$.

Since

$$
\begin{aligned}
H(t+D k) & =\sum_{k \in \mathbb{Z}^{2}} C_{k} e^{-i k^{T}(t+D k)} \\
& =\sum_{k \in \mathbb{Z}^{2}} C_{k} e^{-i k^{T} t} e^{-i k^{T} D k} \\
& \left.=\sum_{k \in \mathbb{Z}^{2}} C_{k} e^{-i k^{T} t} \quad \text { (because } e^{-i k^{T} D k}=1\right) \\
& =H(t),
\end{aligned}
$$

(18) implies that

$$
\begin{aligned}
& \sum_{k \in \mathbb{Z}^{2}} \int_{[0,2 \pi]^{2}}|H(t)|^{2}|\hat{\phi}(t+D k)|^{2} d t \\
& =\int_{[0,2 \pi]^{2}}|H(t)|^{2} \sum_{k \in \mathbb{Z}^{2}}|\hat{\phi}(t+D k)|^{2} d t,
\end{aligned}
$$

where $\sum_{k \in \mathbb{Z}^{2}}|\hat{\phi}(t+D k)|^{2}=\sum_{k \in \mathbb{Z}^{2}}\left|\hat{\phi}\left(\begin{array}{c}t_{1}+2 k_{1} \pi \\ t_{2}+2 k_{2} \pi\end{array}\right)\right|^{2}$. 
Since $\operatorname{supp} \hat{\phi}(\omega)=[-2 \pi, 2 \pi]^{2}$, the $k$ must be chosen by the following value: $k=\left(\begin{array}{l}0 \\ 0\end{array}\right), k=\left(\begin{array}{l}1 \\ 0\end{array}\right), k=\left(\begin{array}{l}0 \\ 1\end{array}\right), k=\left(\begin{array}{l}1 \\ 1\end{array}\right)$.

Then

$$
\begin{aligned}
\sum_{k \in Z^{2}}|\hat{\phi}(t+D k)|^{2} & =\left|\hat{\phi}\left(\begin{array}{c}
t_{1} \\
t_{2}
\end{array}\right)\right|^{2}+\left|\hat{\phi}\left(\begin{array}{c}
t_{1}+2 \pi \\
t_{2}
\end{array}\right)\right|^{2} \\
& +\left|\hat{\phi}\left(\begin{array}{c}
t_{1} \\
t_{2}+2 \pi
\end{array}\right)\right|^{2}+\left|\hat{\phi}\left(\begin{array}{c}
t_{1}+2 \pi \\
t_{2}+2 \pi
\end{array}\right)\right|^{2} \\
& =|\hat{\phi}(t)|^{2}+\left|\hat{\phi}\left(t+\left(D \epsilon_{1}\right)^{T}\right)\right|^{2} \\
& +\left|\hat{\phi}\left(t+\left(D \epsilon_{2}\right)^{T}\right)\right|^{2}+\left|\hat{\phi}\left(t+(D \epsilon)^{T}\right)\right|^{2}
\end{aligned}
$$

where $\epsilon=(1,1)^{T}, \quad \epsilon_{1}=(1,0)^{T}, \epsilon_{2}=(0,1)^{T}$.

Hence (19) means that

$$
\begin{aligned}
& \int_{[0,2 \pi]^{2}}|H(t)|^{2}\left\{|\hat{\phi}(t)|^{2}+\left|\hat{\phi}\left(t+\left(D \epsilon_{1}\right)^{T}\right)\right|^{2}\right. \\
& \left.+\left|\hat{\phi}\left(t+\left(D \epsilon_{2}\right)^{T}\right)\right|^{2}+\left|\hat{\phi}\left(t+(D \epsilon)^{T}\right)\right|^{2}\right\} d t .
\end{aligned}
$$

We now denote the parenthetical part of the integrand in equation (20) by $\Omega=$ $|\hat{\phi}(t)|^{2}+\left|\hat{\phi}\left(t+\left(D \epsilon_{1}\right)^{T}\right)\right|^{2}+\left|\hat{\phi}\left(t+\left(D \epsilon_{2}\right)^{T}\right)\right|^{2}+\left|\hat{\phi}\left(t+(D \epsilon)^{T}\right)\right|^{2}$. Since the Fourier transform $\hat{\phi}(w)$ is continuous and $\operatorname{supp} \hat{\phi}(w)=[-2 \pi, 2 \pi]^{2}, \Omega$ clearly has the Riesz positive bounds $\frac{1}{A}$ and $\frac{1}{B}$.

Therefore, we can write

$$
\frac{1}{A} \geq \sup _{t \in[0,2 \pi]^{2}}\{\Omega\}, \quad \frac{1}{B} \leq \inf _{t \in[0,2 \pi]^{2}}\{\Omega\} .
$$

Thus we have

$$
\begin{aligned}
\frac{1}{A} \int_{[0,2 \pi]^{2}}|H(t)|^{2} d t & \geq \int_{[0,2 \pi]^{2}}|H(t)|^{2}\{\Omega\} d t \\
& =\int_{[0,2 \pi]^{2}}|\hat{u}(w)|^{2} d w=\|\left. u\right|^{2} \\
\frac{1}{B} \int_{[0,2 \pi]^{2}}|H(t)|^{2} d t & \leq \int_{[0,2 \pi]^{2}}|H(t)|^{2}\{\Omega\} d t \\
& =\int_{[0,2 \pi]^{2}}|\hat{u}(w)|^{2} d w=\|u\|^{2} .
\end{aligned}
$$

By combining (21) and (22), we have

$$
\begin{aligned}
B^{-1} \int_{[0,2 \pi]^{2}}|H(t)|^{2} d t & \leq \int_{\mathbb{R}^{2}}|\hat{u}(w)| d w \\
& \leq A^{-1} \int_{[0,2 \pi]^{2}}|H(t)|^{2} d t
\end{aligned}
$$


Evaluating the inequalities of (23) yields

$$
\begin{aligned}
& 2 \pi \sum_{k \in \mathbb{Z}^{2}}\left|C_{k}\right|^{2}=\int_{[0,2 \pi]^{2}}|H(t)|^{2} d t \geq A \int_{\mathbb{R}^{2}}|\hat{u}(w)|^{2} d w=A\|u\|^{2} \\
& 2 \pi \sum_{k \in \mathbb{Z}^{2}}\left|C_{k}\right|^{2}=\int_{[0,2 \pi]^{2}}|H(t)|^{2} d t \leq B \int_{\mathbb{R}^{2}}|\hat{u}(w)|^{2} d w=B\|u\|^{2}
\end{aligned}
$$

and thus we have $A\|u\|_{L^{2}\left(\mathbb{R}^{2}\right)}^{2} \leq \sum_{k \in \mathbb{Z}^{2}}\left|C_{k}\right|^{2} \leq B\|u\|_{L^{2}\left(\mathbb{R}^{2}\right)}^{2}$.

Hence, we obtain that $\phi(x-k), k \in \mathbb{Z}^{2}$, is a Riesz basis of $V_{0}$, which completes the proof.

Now, by using the same vein of the one-variable wavelet analysis, the two-variable wavelet functions can be described efficiently akin to the version of the tensor product $L^{2}(\mathbb{R}) \otimes L^{2}(\mathbb{R})$.

Let us set

$$
h(2 x-y)=h_{1}\left(2 x_{1}-y_{1}\right) h_{1}\left(2 x_{2}-y_{2}\right) .
$$

If we let $\operatorname{supph} \hat{h_{1}}(w)=[-\pi, \pi]$, then

$$
\begin{aligned}
& \int_{\mathbb{R}} \int_{\mathbb{R}}\left\{h_{1}\left(2 x_{1}-y_{1}\right) h_{1}\left(2 x_{2}-y_{2}\right)\right\}^{2} d y_{1} d y_{2} \\
& =\int_{\mathbb{R}}\left\{h_{1}\left(2 x_{1}-y_{1}\right)\right\}^{2} d y_{1} \int_{\mathbb{R}}\left\{h_{1}\left(2 x_{2}-y_{2}\right)\right\}^{2} d y_{2}
\end{aligned}
$$

where $h_{1}\left(2 x_{1}-y_{1}\right) h_{1}\left(2 x_{2}-y_{2}\right) \in L^{2}(\mathbb{R}) \bigotimes L^{2}(\mathbb{R})\left(\subset L^{2}\left(\mathbb{R}^{2}\right)\right)$.

From these descriptions we readily obtain the following theorem constructing two-variable multiresolution analysis:

Theorem 3.4. If $\phi(x)$ is the solution of the equation $\phi(x)=\lambda \int_{\mathbb{R}} h(2 x-y) \phi(y) d y$ and supph $\hat{h}(w)=[-\pi, \pi]$, then $V_{j}=\operatorname{span}\left\{\phi\left(2^{j} x_{1}-k_{1}\right) \phi\left(2^{j} x_{2}-k_{2}\right) \mid k_{1}, k_{2} \in \mathbb{Z}\right\}$ constructs a two-variable multiresolution analysis.

\section{REFERENCES}

[1] J. Aguirre, M. Escobedo, J.C. Peral, and P.H. Tchamitchian, Basis of wavelets and atomic decompositions of $H^{1}\left(\mathbb{R}^{n}\right)$ and $H^{1}\left(\mathbb{R}^{n} \times \mathbb{R}^{n}\right)$, Proceedings AMS 111 (1991), 683-693. MR 91k:42037

[2] P. G. Casazza and A.O. Christensen, Frames containing a Riesz basis and preservation of this property under perturbations, SIAM J. Math. Anal. 29 (1998), 266-278. MR 99i:42043

[3] C. K. Chui, An Introduction to Wavelets, Academic Press, Inc (1992). MR 93f:42055

[4] M. G.Cui, D. M. Lee, and J. G. Lee, Fourier Transforms and Wavelet Analysis, Kyung Moon Press (2001).

[5] R. Coifman, and Y. Meyer, Remarques sur L'analyse de Fourier a fenetre, C. R. Acad. Sci. pairs t.312 (1991), 259-261. MR 92k:42042

[6] I. Daubechies and J. C. Lagarias, Two-Scale Difference Equations I, Existence and global regularity of Solutions, SIAM J. Math. Anal. 22 (1991), 1388-1410. MR 92d:39001

[7] I. Daubechies, Ten Lectures on Wavelets, SIAM, Philadelphia, PA (1992). MR 93e:42045

[8] K. Grochenig and W. R. Madych, Multiresolution Analysis, Haar Bases, and Self-Similar Tilings of Rn, IEEE Trans. on Information Theory 38 (1992), 556-568. MR 93i:42001

[9] A. Grossmann and J. Morlet, Transforms associated to square integrable group representations I, J. Math. Phys. 26 (1986), 2473-2479. MR 86k:22013

[10] B. Jawerth and W. Sweldens, An overview of wavelet based multiresolution analysis, SIAM Rev. 36(3) (1994), 377-412. MR 95f:42002

[11] W. Lawton, Necessary and sufficient conditions for constructing orthonormal wavelet bases, J. Math. Phys. 32 (1) (1991), 57-61. MR 91m:81100 
[12] J. G. Lee and D. M. Lee, A Filtering Formula on Wavelets, Korean Annales of Math. 15 (1998), 247-255.

[13] S. Mallat, A theory for multiresolution signal decomposition: the wavelet representation, IEEE Trans. Pattern Anal. Machine Intell. 11 (1989).

College of Mathematics Science, Won Kwang University, 344-2 Shinyongdong Ik-San, Chunbuk 570-749, Korea

E-mail address: dmlee@wonkwang.ac.kr

College of Mathematics Science, Won Kwang University, 344-2 Shinyongdong Ik-San, Chunbuk 570-749, Korea

College of Mathematics Science, Won Kwang University, 344-2 Shinyongdong Ik-San, Chunbuk 570-749, Korea 